\title{
A Morphing Radiator for High-Turndown Thermal Control of Crewed Space Exploration Vehicles
}

\author{
Thomas J. Cognata ${ }^{1}$ \\ Paragon Space Development Corporation, Houston, TX, 77058 \\ Darren J. Hartl $1^{2}$ \\ Texas Institute for Intelligent Materials and Structures, Texas A\&M University, \\ College Station, TX, 77845-3409, USA \\ Rubik Sheth ${ }^{3}$, Craig Dinsmore ${ }^{4}$ \\ National Aeronautics and Space Administration, \\ Lyndon B. Johnson Space Center, Houston, TX, 77058
}

\begin{abstract}
Spacecraft designed for missions beyond low earth orbit (LEO) face a difficult thermal control challenge, particularly in the case of crewed vehicles where the thermal control system (TCS) must maintain a relatively constant internal environment temperature despite a vastly varying external thermal environment and despite heat rejection needs that are contrary to the potential of the environment. A thermal control system may be required to reject a higher heat load to warm environments and a lower heat load to cold environments, necessitating a relatively high turndown ratio. A modern thermal control system is capable of a turndown ratio of on the order of 12:1, but crew safety and environment compatibility have constrained these solutions to massive multi-loop fluid systems. This paper discusses the analysis of a unique radiator design that employs the behavior of shape memory alloys (SMAs) to vary the turndown of, and thus enable, a single-loop vehicle thermal control system for space exploration vehicles. This design, a morphing radiator, varies its shape in response to facesheet temperature to control view of space and primary surface emissivity. Because temperature dependence is inherent to SMA behavior, the design requires no accommodation for control, instrumentation, or power supply in order to operate. Thermal and radiation modeling of the morphing radiator predict a turndown ranging from 11.9:1 to 35:1 independent of TCS configuration. Coupled thermal-stress analyses predict that the desired morphing behavior of the concept is attainable. A system level mass analysis shows that by enabling a single loop architecture this design could reduce the TCS mass by between $139 \mathrm{~kg}$ and $225 \mathrm{~kg}$. The concept has been demonstrated in proof-of-concept benchtop tests.
\end{abstract}

\footnotetext{
${ }^{1}$ Insert Job Title, Department Name, Address/Mail Stop, and AIAA Member Grade for first author.

${ }^{2}$ Insert Job Title, Department Name, Address/Mail Stop, and AIAA Member Grade for third author.

${ }^{3}$ Insert Job Title, Department Name, Address/Mail Stop, and AIAA Member Grade for fourth author (etc).

${ }^{4}$ Insert Job Title, Department Name, Address/Mail Stop, and AIAA Member Grade for fourth author (etc).
} 


\section{Extended Abstract (Note: SciTech requires a 500 word min extended abstract)}

Spacecraft designed for missions beyond low earth orbit (LEO) face a difficult thermal control challenge, particularly in the case of crewed vehicles. The thermal control system must maintain a relatively constant internal environment temperature despite a vastly varying external thermal environment, which may present a sink temperature that ranges from $70 \mathrm{~K}$ in transplanetary coast (TPC) to approximately $228 \mathrm{~K}$ in planetary surface operations (PSO), and heat rejection needs that are contrary to the change in capacity of the environment. In other words, the thermal control system (TCS) is required to reject a higher heat load to warm environments and a lower heat load to cold environments, necessitating a quite high turndown ratio.

This thermal design need is complicated by the challenge of transporting heat from

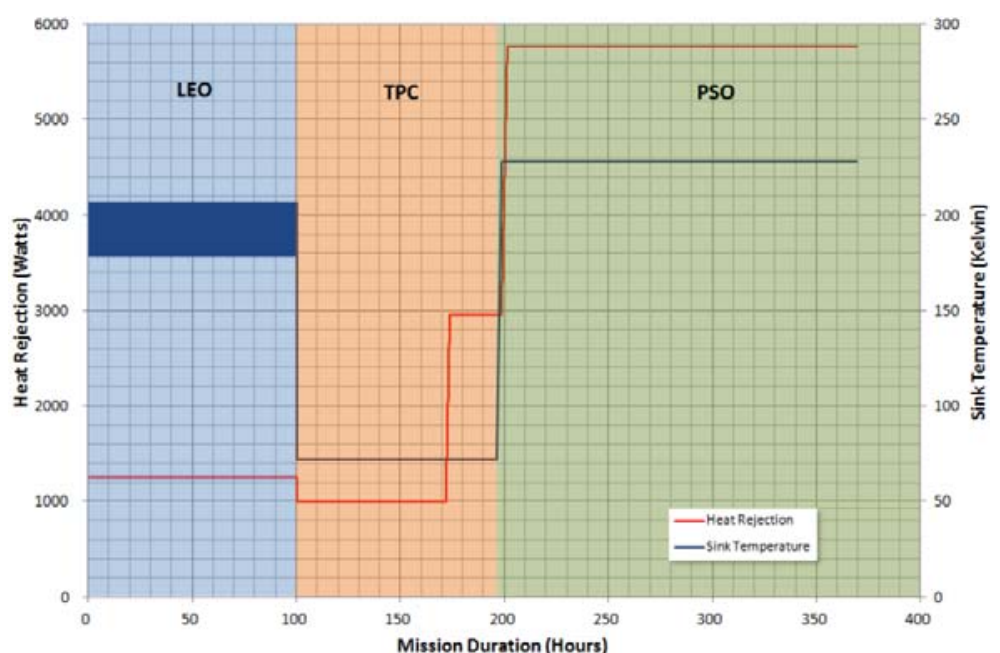

Figure 1 A representative mission profile illustrating varying thermal environment and heat rejection.

crewed portions of the vehicle to the heat rejection portion of a TCS without the endangerment of crewmembers due to working fluid toxicity or any potential reaction of the working fluid with other vehicle systems as a consequence of a leak. This drives the current state of the art TCS design - a two-loop system with a propylene-glycol/water working fluid contained in an inner TCS loop and with a low freezing point refrigerant in a loop wholly external to the cabin which is able to operate over the very wide range of temperatures that the radiator is subject to. While conservative, this design has the drawback of adding significant mass and complexity to the TCS, interfacial inefficiencies to heat transfer between the loops, and the duplication of fluid handling equipment such as pumps and expansion tanks. Previous trades have shown that a two-loop TCS is approximately $25 \%$ heavier than a similarly performing single loop system.

Electrochromics, heat pumps, loop heat pipes, and stagnating radiators are among several technologies being actively investigated toward the adoption of a single loop TCS, but each has shortcomings that either severly limit the turndown possible to little better than $4: 1$ or add considerable launch mass and additional heat load. The use of shape memory alloys (SMA) for temperature dependent variable heat rejection is a transformative development in the field and promises the theoretical potential to meet mission turn-downs as great as 60:1 in the environment described by Figure 1.

Shape memory alloys are a category of metals with unique properties derived from a reversible, temperature dependent, solid-state phase transformation. The phase transition is between the austenitic phase, which is stable above a transition temperature and the martensitic phase, which is stable below a different (colder) temperature. It is the temperature dependence of this transition, the large deformation afforded by the martensitic phase, and the large working loads that make the shape memory alloy an attractive thermal actuator for high turn-down control of spacecraft heat rejection. The material's unique temperature dependent behavior provides a key advantage

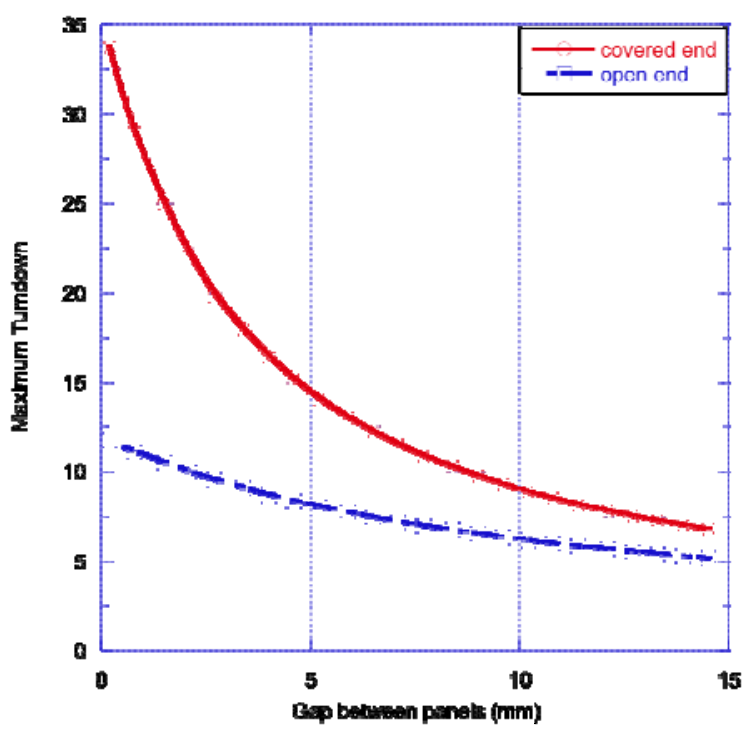

Figure 2 Sensitivity to gap in the cold case. Shows sensitivity of turndown to the gap between the end of each side of the facesheet for this concept, with and without end shields. that design requires no accommodation for control, instrumentation, or power supply in order to operate. 
A first order estimation of the turn-down possible based on the definition of radiation heat rejection indicates the potential for a $60: 1$ is turndown when taking into account the target environment of Figure 1.

Finite element analysis in a representative environment indicates that the design turndown in fact approaches 35.2:1. The difference relative to the analytical prediction is a consequence of the assumption of a constant surface temperature in the latter case.

A finite element based optimization study further finds that any gap between the surfaces of the facesheet when in the cold shape has an influence on the maximum turndown. Figure 2 shows the turndown from a perfect cold shape, or $0 \mathrm{~mm}$ gap, to $15 \mathrm{~mm}$ (0.59 in) gap between faces. Further, as seen in Figure 3, turndown is not strongly sensitive to a radius of curvature in the hot shape once greater than $\sim 0.1 \mathrm{~m}$. These result indicate a focus in later design on cold shape, as opposed to hot shape, fidelity

A coupled thermal-stress finite element analysis shows the facesheen to open substantially, with the majority of the flattening deformation induced by the SMA concentrated near the flow tube heat source, as expected. This can be seen in Figure 4. The contours in

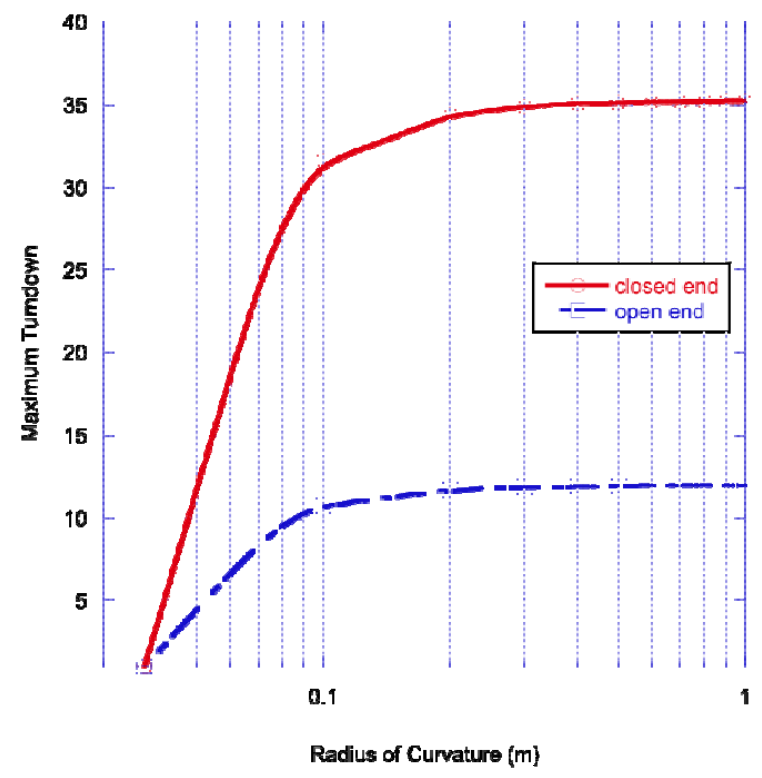

Figure 3 Sensitivity to curvature for the hot case. Shows sensitivity in turndown to the hot case curvature of the facesheet. A curvature of 0.1 is approximately a half circle. this figure show the axial stress in the glass fiber laminae transverse to the fiber direction, which corresponds to the largest stress component induced by the bending. The limits of the contour legend are equivalent to the calculated values for axial stresses at failure in this direction.
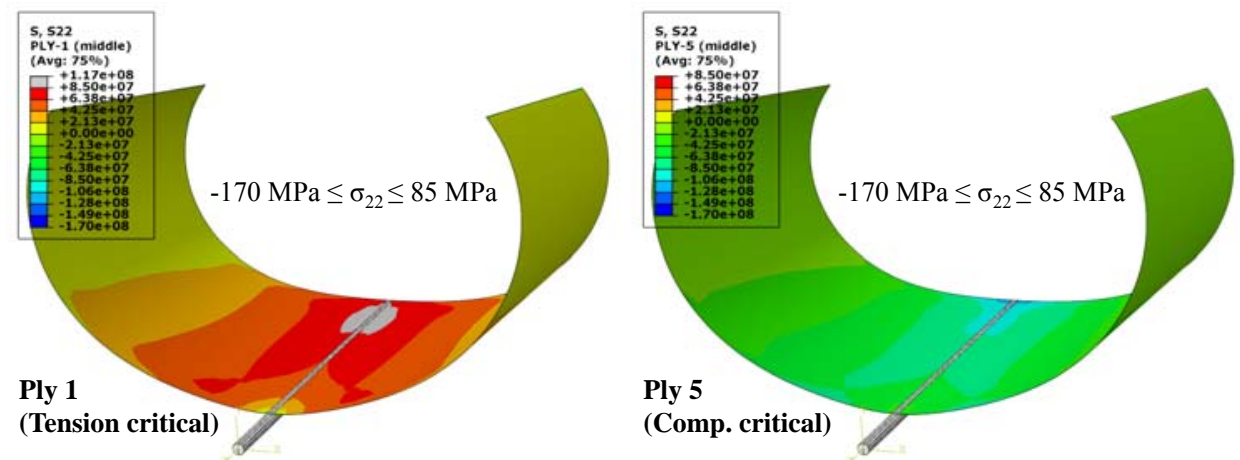

\section{Figure 4 - Global heated deformation and local stress results (S22) in the glass fiber-based laminae (contour plot bounds correspond to stress limits).}

The high turn-down of this technology permits radiator operating temperatures that enable a single fluid-loop TCS using a human compatible fluid such as a propylene glycol/water mixture. In terms of mass impact, for a $5800 \mathrm{~W}$ peak heat rejection an Orion based architechture, this design therefore has the potential to reduce launch mass by between $139 \mathrm{~kg}$ and $225 \mathrm{~kg}$. This reduction benefits largely through the savings of a single-loop architecture. 